$\xi_{p}$

\title{
An Analysis of Intellectual Property Challenges in Crowdsourcing Platforms for Software Engineering
}

\author{
Hani Al-Bloush ${ }^{1}$, Badariah Solemon ${ }^{2 *}$ \\ ${ }^{1}$ College of Graduate Studies, Universiti Tenaga Nasional \\ ${ }^{2}$ College of Computer Science and Information Technology, Universiti Tenaga Nasional \\ *Corresponding authorE-mail: badariah@uniten.edu.my
}

\begin{abstract}
Crowdsourced Software Engineering (CSE) is an application of online problem-solving model that provides a dynamic way of harnessing crowd intelligence in obtaining creative solutions. It consists of four elements: crowdsourcer, crowd, platform, and tasks. Although CSE offers various advantages to software engineering practices, recent research highlighted that there is still a lack of thorough understanding of Intellectual Property (IP) ownership rights of CSE elements. Therefore, management and control of CSE for obtaining adequate IP from the crowd is inevitable to reduce the risks of using that IP. This paper reviews existing CSE platforms and analyses associated IP challenges encountered by software engineering activities. 51 platforms were preselected and amongst these, analyses were performed on $37 \mathrm{CSE}$ platforms. Exclusion of 11 platforms is on the basis of unavailability and 3 of which are linked to the same webpage. It also shows that $59 \%$ of the platforms acquire IP ownership of deliverables submitted by the crowd while $19 \%$ do not have a statement which provides clarity on IP ownership in their legal documents. This paper is significant in providing better understanding of IP risks of these CSE platforms and in assisting both crowdsourcers and the crowd in choosing crowdsourcing platforms based on the default IP ownership specification, which is often specified in legal documents of these platforms.
\end{abstract}

Keywords: Crowdsourced Software Engineering (CSE); Intellectual Property (IP); IP ownership; platform.

\section{Introduction}

Crowdsourcing today has obtained increasing demand because of its ability to delegate a variety of tasks which are requested by individuals, institutions, and organizations to an unknown workforce who is able to accomplish the tasks. These requested tasks from different areas are usually assigned by means of an open call format through a variety of online platforms [1]. In June 2006, the term 'crowdsourcing' was first introduced by Jeff Howe and Mark Robinson in Wired magazine [2] as "the act of a company or institution taking a function once performed by employees and outsourcing it to an undefined (and generally large) network of people in the form of an open call. This can take the form of peerproduction (when the job is performed collaboratively), but is also often undertaken by sole individuals. The crucial prerequisite is the use of the open call format and the large network of potential laborers". Crowdsourcing utilizes the so-called "collective intelligence' of the crowd to provide creative solutions through an online open call to serve a specific task. The conditions for crowdsourcing as stated in the aforementioned definition is typically given by online crowdsourcing platforms, which in turn allows crowd participants of considerable number to provide resolutions for specific crowdsourcers' problems, difficulties, or goals. There are four elements of a crowdsourcing approach as shown in Figure1: the crowdsourcer (also known as requesters), the crowdsourcing platform, the crowd (also known as worker or participants), and the crowdsourced tasks.

Crowdsourcing has been applied in various domains such as software development, education, news, management, and economics
[3-5]. Instances of crowdsourcing applications include hazardous weather reports by the residents using social media Twitter [6], design and sales of T-shirts [7,8], creation of crowdsourced textbook and other course or class materials of higher education [9] and programming competition of the SPHERES Zero Robotics [10]. The utilization of crowdsourcing platforms resulted in the decrease of overhead expenses, reduction of time, and increase in flexibility and convenience of performing tasks for individuals, institutions, and organizations. Among the known crowdsourcing strategies include crowd creation, crowd wisdom, crowd funding, and crowd voting as explained in $[11,12]$.

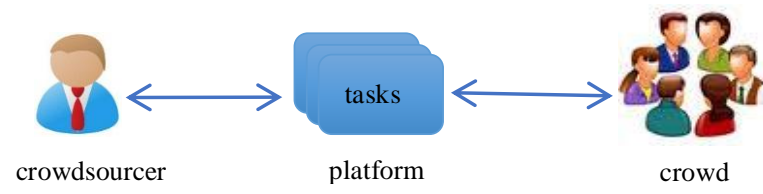

Fig. 1: The elements of a crowdsourcing approach

At present, one notable variation of the generic crowdsourcing approach with a remarkable status in the open collective intelligence environment is known as Crowdsourced Software Engineering or CSE in short. As mentioned by Archak [3], CSE that is derived from the general principle of the crowdsourcing approach, allows software development professionals to be recruited from a large pool of crowds to accomplish software engineering tasks posted on online crowdsourcing platforms. In CSE software development professional may get access for different tasks from online crowdsourcing platforms including TopCoder (TC) [13], Amazon Mechanical Turk (AMT) [14], and 99Test [1, 14] without having to be permanently employed by the organizations. It is 
reported in literature that the application of CSE reduces the software crisis particularly time-to-market, increases software and software components' quality, and minimizes costs through the submission of tasks to a group of crowd participants with flexible development ability $[15,16]$. Also, CSE success is fundamental in attracting the crowdsourcers to allure the crowds to perform various software tasks and vice versa and most importantly in significantly increasing the creation of online crowdsourcing platforms. Despite these advantages, recent research studies highlighted that the application of CSE raises legal issues related to Intellectual Property (IP) risks [1,17]. IP is defined in [18] as "someone's idea, invention, creation, etc., that can be protected by law from being copied by someone else". IP also refers to the multi-ownership intangible rights, for example, creation or product of the human mind [19]. Within the context of online crowdsourcing platforms for software engineering, apparently, there is still a lack of thorough understanding with regards to the ownership of IP rights. Therefore, management and control of CSE in obtaining adequate IP from the crowd became inevitable to reduce the risks of using that IP. Consequently, the research presented in this paper aims to achieve two main objectives. The first objective aims at identifying the CSE platforms supporting software engineering practices. While the second objective aims at investigating IP challenges in those identified CSE platforms and at revealing the extent of these challenges. In order to achieve the two research objectives, a set of Research Questions (RQ) is formed in this research as follows:

- RQ1: What are the crowdsourcing platforms that support CSE activities?

- RQ2: When did the platforms emerge in the market?

- RQ3: Who owns the IPs of the contributions by the crowd?

- RQ4: What are the challenges related to IP ownership rights and the extent of these challenges faced by CSE platforms identified in RQ1?

The remaining part of this paper is structured as follows: Section 2 presents the literarture review of related work of this study, while Section 3 illustrates the methodology that was used in conducting the research explained in this paper. In Section 4, the results obtained are presented and discussed and lastly, in Section 5, we conclude by offering recommendations for managing and controlling IP in deliverables.

\section{Related Work}

As mentioned earlier, the crowdsourcer, the crowdsourcing platform, and the crowdsourced tasks are the three components which are of particular focus of researches conducted regarding crowdsourcing $[1,20]$ while inadequate consideration was allotted to the IP concerns and challenges arising within the components of CSE. A number of studies have contemplated the challenges of CSE including the extent to which these challenges have influenced the crowdsourcing activities. Also, several other studies [1, 7, 10, 21 , 22 - 23] have been acknowledged a variety of CSE challenges including IP and data security (e.g confidentiality) issues. Some of these issues regarding IP and data security include: (i) the dangerous risks in allocating tasks without long and careful consideration of IP and confidentiality information, involving privacy and security risks in order to obtain permission to utilize crowdsourced content from the right crowd participants [24], (ii) the concern about IP ownership that focuses on who would actually own the IP of tasks created by the crowd either the the crowdsourcer, the crowdsourcing platform, the crowd, or combination of the three crowdsourcing elements [25], (iii) the risk of retaining knowledge in the crowd [26] which occurs once the crowdsourcing tasks are transferred to the crowd and after the tasks are completed, a behaviour which cannot be prevented and become worse in the context of for-profit crowdsourcing, and (iv) the possible violation of third-party or pre-existing content $[1,27]$ in the completed crowdsourcing tasks. For instance, it is possible that the crowd might submit third-party content prepared for non-commercial purposes despite the crowdsourcer's requests for commercial intention. In another instance, the crowd might submit tasks created from Open Source Software (OSS), which will make the tasks be subjected to OSS governing license $[1,17]$.

As suggested in [28, 29], the risk of losing IP and IP management and control should be considered. Similarly, the three issues of IP 1) the loss of IP ownership , 2) IP 'leakage' and 3) data security are also stated specifically in the book of "Software Sourcing in the Age of Open" [30] with IP loss being ranked as the most significant threat in CSE practices.

While existing literature emphasizes concerns about IP risks in general and how to balance ownership rights among the crowd, the crowdsourcer and the CSE platform, there has been no investigation conducted on how platforms deal with IP rights. We build on this by providing an analysis of legal documents and listing out the available IP ownership options that platforms consider when crowdsourcing CSE solutions.

\section{Methodology}

In this study, a 'multi-phase' approach was applied in order to determine the software engineering platforms and to analyse the status of IP ownership within the legal documents of these online platforms. The legal documents are often named 'terms and conditions' but may also be called as 'terms of use', 'terms of service', 'terms and privacy', 'privacy policy', 'policy', 'copyright infringement policy', 'legal', 'legal terms', 'user agreement', and 'participation agreement'. An example of screenshot of the legal document reviewed in this research, the 'Terms and Conditions' of one of the platforms, is as shown in Figure 2.

The analysis to be presented in this paper was performed based on whether the crowdsourcing platforms provide the appropriate legal documents which shall define the relationship between the platform's users and their IP ownership rights. There are five phases in the methodology of the study as shown in Figure 3. By reviewing scholarly contributions related to crowdsourcing challenges within the domain of software engineering, several CSE platforms are identified and their IP ownership issues and challenges are examined.

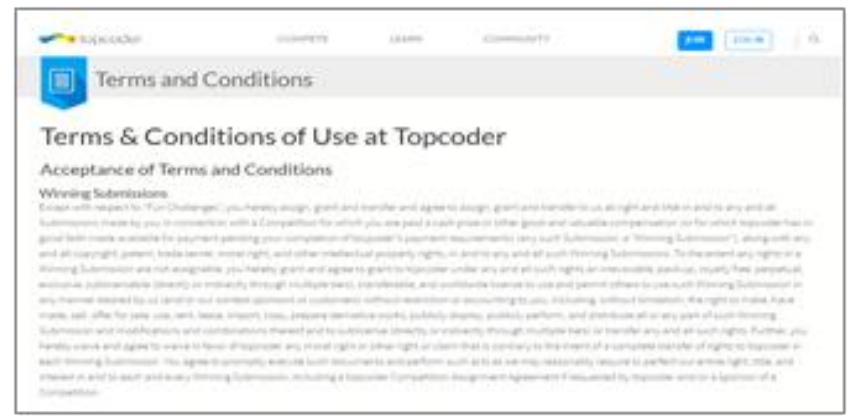

Fig. 2: Sample of a legal document of the platform [1]

The methodology of the study has been effectively applied in another study explained in [21], which determines the factors affecting the decision to crowdsource. Similar to the adopted methodology are also described in other studies [31-33], the five phases are briefly explained below:

- $\quad$ Phase I - Identification of RQs: In this phase, research questions was formulated to guide and determine the direction of this study, including the identification of generic keyword to perform the subsequent search procedure. The generic keyword used is "crowdsourced software engineering".

- $\quad$ Phase II - Search Online Database: In this phase, the general keyword identified in Phase I was used to search selected online databases. The online databases selected in this study include 1) ACM Digital library, 2) IEEExplore, 3) ScienceDirect, and 4) Scopus, which are subscribed by the 
library of UNITEN. This phase managed to identify 22 relevant documents from these databases. The identified documents were scanned by evaluating their relevance to software engineering activities, crowdsourcing platforms and intellectual property in order to discover existing IP challenges within the context of CSE practices.

- $\quad$ Phase III - Identify CSE Platforms and IP Challenges: This phase produces a list of CSE platforms and challenges faced by the platforms from the results of analysis performed to the legal documents of the platforms with respect to the status of IP ownership rights.

- $\quad$ Phase IV - Evaluate Initial Validity. This phase evaluates the validity of each CSE platform based on three validation criteria. The criteria are as follows: 1) Availability and accessibility of the platform to the public, 2) Existence of IP rights legal documents in the platform, 3) Existence of IP ownership statements and related agreements or instructions in the platform. Only platforms that are available, accessible, and dealing with IP ownership right are analysed to clearly identify the key IP ownership issues and the variation of the legal documents.

- $\quad$ Phase V - Report Findings: Preparation of research findings report. The research findings are concluded in this last phase after a few rounds of Phase II, III and IV.

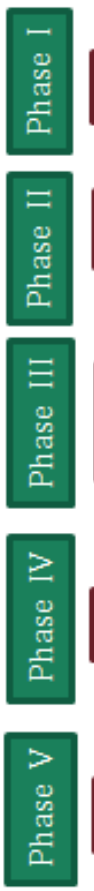

Identification of RQs

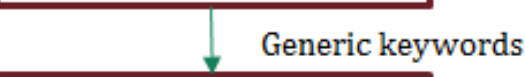

Search Online Database

List of relevant articles

Identify CSE Platforms and IP Challenges

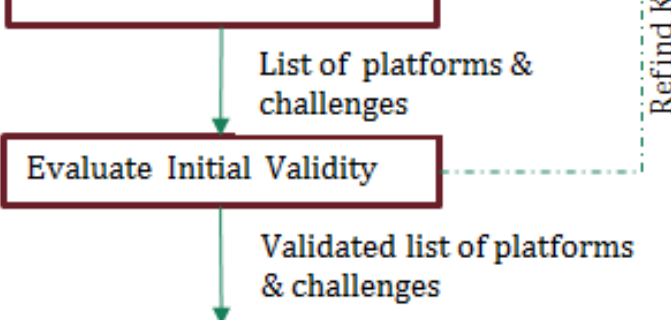

Report Findings

Fig. 3: The methodology of the study

\section{Results and Discussion}

This section presents and discusses the results that provide answers to the above-mentioned RQs. The section begins with the the list of identified crowdsourcing platforms and followed by analysis of the establishment and accessibility of the platforms. Subsequently, the timeline of the platforms are analysed. From the analysis of the legal documents of CSE platforms, observations are derived and from the review of existing literature with regards to CSE practices, the challenges of IP are determined.

\subsection{CSE Platforms}

A total of 51 online crowdsourcing platforms that support software engineering activities have been identified from the review performed to 22 relevant articles in the literature. Appendix 1 summarizes the name, URL and references of the platforms. Each crowdsourcing platform is also assigned with a unique ID number labelled from P1 to P51 to identify the platforms in the analysis. The online platforms can be classified generally into two categories: 1) Exclusive software engineering crowdsourcing platforms [34] and 2) Partial software engineering platforms [25]. TopCoder (P1) and Innocentive (P3) are examples of online crowdsourcing developed to support exclusively software engineering activities. On the other hand, AMT (P25) [35], Upwork (P24), and Freelancer (P22) [36] are examples of online crowdsourcing platforms that can still be used to support various activities of the software engineering despite they were not designed for software engineering activities. Appendix 1 also lists the specific primary work category of each platform. The specific primary work categories of the crowdsourcing platforms are project marketplace (9), software testing (9), software development (8), graphic design (5), problem solving (3), mobile app testing (3), software security testing (3), programmer marketplace (3), 'question and answer' (2), 'any task' (2), mobile development (1), technical support (1), and data mining (1). These results provide answer to RQ1: What are the crowdsourcing platforms that support CSE activities?

\subsection{Timeline of CSE Platforms}

Timeline analysis is a useful process for a variety of investigation types and is often used to answer questions about when or what events occurred before or after a particular time [37, 38]. No information was found for 6 out of 51 platforms: Tackcn (P23), Freelance Web Programming (P38), Programming bids (P40), Programmermeet Desiner.com (P44), Project for HIREFreelance Marketplace (P45) and Zintro (P50). Thus, the remaining 45 CSE

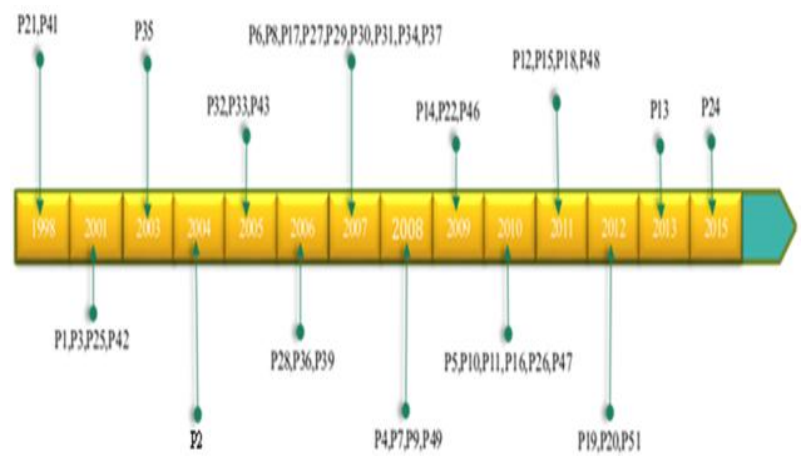

Fig. 4: Timeline diagram of the establishment of CSE platforms

platforms are analysed in this study using a timeline diagram as shown in Figure 4. Unfortunately, at the time of writing this article, another six platforms GetACoder (P2), CrowdTesters (P15), TestFlight (P16), Testin (P18), Ce.WooYun (P19), AskvilleAmazon (P28), CGILance.com (P35), LiveWork (P46), and AppStori (P51) are also no longer accessible by the public. Five of these platforms (P2, P15, P16, P18 and P19) have been reported in $[17,34]$. Interesting enough, a research website founded by users of Amazon.com known as Askville-Amaz (P28) was reported as available in $[21,39]$ despite the fact that it was shut down in 2013. Similarly, Tacken (P23) was reported in $[3,40]$, but there is no longer a website that supports the platform.

As can be seen from Figure 4, the number of CSE platforms' establishment varies from one year to another. From 2006 to 2012, there was a marked increase in the number of CSE platforms in 2007 and 2010 after the acceptance of the crowdsourcing as a solution model via online open call format in 2007. As for the platforms established before 2006, these were earlier purely online shopping and project marketplace platforms such as AMT (P25) [41] and Rent A Coder (P41), but recently they have been added with crowdsourcing tasks. The analysis performed to the timeline of the 45 platforms provide answer to RQ2: When did the platforms emerge in the market?

Within the context of software engineering, the rising number of CSE platforms indicates the successful shift of software development activities from small isolated group to an online open pool of 
software engineering professionals who work together, building state-of-the-art solutions and bringing down the development cost [1, 42]. Despite the encouraging trend, there is still a range of challenges and obstacles facing the practice of CSE that must be addressed. Review of the literature [1, 17, 21, 25, 30, 42] disclosed a number of these challenges including task breakdown, quality assurance, management and interaction, incentive and remunerations, intellectual property and data security. Consequently, these challenges have a direct impact on the practices of crowdsourcing by affecting the attractiveness of crowdsourcing and its role in software engineering domain.

\subsection{IP ownership of CSE Platform}

As previously mentioned, 51 platforms in relation to CSE activities are identified. Out of these, 37 platforms are available and accessible for further analysis. These platforms provide services to worldwide users in terms of both generic crowdsourcing activities and specific software engineering crowdsourcing activities as well Also, the review of the legal documents of these online crowdsourcing platforms result in the grouping of the platforms into four main categories, which are formed based on the ownership of the IP created by the crowd of the crowdsourcing tasks or content: 1) the crowdsourcer (CSE requesters), 2) the crowd (workers or participants), 3) the online platforms (crowdsourcing platforms or service providers) or 4) none of the above. The last category is created to indicate the lack of direct or indirect statement of who should have the IP ownership rights transacted in the online platforms). Table 1 presents the distribution of IP ownership rights of the CSE platforms, based on those four categories, which provides answer to RQ3: Who owns the IPs of these platforms?

\begin{tabular}{|c|c|}
\multicolumn{2}{|c|}{ Table 1: Classification of CSE platforms } \\
\begin{tabular}{|c|c|}
\hline Category & Platforms \\
\hline Crowd & P4, P7, P24, P26, P29, P45, P47 \\
\hline Crowdsourcer & P3, P12 \\
\hline Online Platform & P1, P5, P8, P9, P10, P11, P14, P17, P20, P22, \\
& P25, P27, P30, P31, P32, P33, P34, P37, P40, \\
& P48, P49, P50 \\
\hline Unknown & P6, P13, P21, P36, P43, P44 \\
\hline
\end{tabular}
\end{tabular}

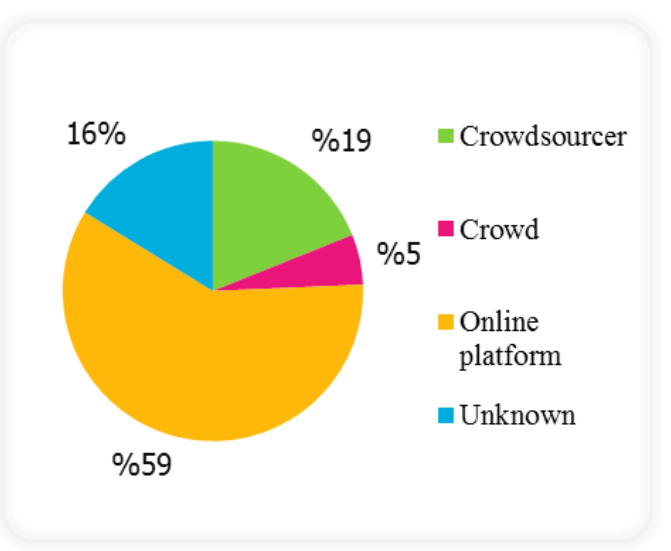

Fig. 5: IP ownership of CSE platforms

Figure 5 presents that $59 \%$ of online crowdsourcing platforms are observed to be granted with the IP ownership rights for all CSE contents submitted by the crowd participants. Seven or $19 \%$ of these CSE platforms directly assign, grant and transfer the actual IP ownership rights of content submitted by crowd participants to the crowdsourcers, while another seven platforms do not have a clear statement regarding on who is the actual owner of the IP ownership rights. The remaining $5 \%$ of the platforms provides the liberty to the participating entities the decision on the IP ownership rights. This can be concluded through an agreement with the crowdsourcers whether to retain IP ownership rights, to transfer the ownership, or to exploit the submitted content for financial or non-financial purposes.

\subsection{Challenges of IP Ownership Rights}

The platforms were also analysed to find answers to the final research question RQ4: What are the challenges related to IP ownership rights and the extent of these challenges faced by CSE platforms identified in RQ1? Amongst the findings are as described in this section. The analysis shows that four out of 37 available and accessible platforms Eufreelance (P37), Limeexchange (P39), Rent A Coder (P41), and Scriptlance (P42) are actually linked to the same website known as the "freelancer.com". Meanwhile, at the time of writing this paper, eleven platforms (P2, P15, P16, P18, P19, P23, P28, P35, P38, P46, and P51) are no longer accessible to the public

The abovementioned results provides clear evidence that there is IP ownership and protection issues especially in managing and controling the IP content of the CSE platforms. This is based on the fact that majority of the CSE platforms grant themselves the IP ownership rights, which contradicts with the goal of online crowdsourcing platforms that is supposed to be a facilitator, mediator, and coordinator between the crowdsourcer and the crowd. These waivers do not offer any support to the crowdsourcers if application of any kind of IP rights for secure protection such as patent, copyright, or trademark is required. However, in order to deprive the crowds of any IP rights and to prevent any future claims and transfer of the deliverables to be a subject of public use, exchange for monetary rewards or other recognitions is done. In addition, a percentage of these platforms allows for assignment and grants of all deliverables (completed crowdsourcing tasks) directly to the crowdsourcers without taking into consideration whether the work is original and not a part of any existing work (which may cause infringing of third-party rights). As for the platforms that allow direct communication between crowdsourcers and crowds, although it is only $5 \%$, it is a good step that should not be limited to assign and grant only, but must include guarantees and contracts that ensures the credibility of productive work and to avoid any future claims that can cost a lot of fines and probability of imprisonment. In addition, the results suggest that quite a few platforms need to establish appropriate legal documents that can serve all their users.

According to the World Intellectual Property Organization (WIPO), although may differ from one country to another, generally the actual IP created by the crowd participants shall be granted to the crowdsourcer, and not the online crowdsourcing platform [42]. As observed and agreed upon by other studies [1, $16,17,25]$, the software engineering activities demands a high degree of creativity. In addition, there is no available guideline or standard in governing IP ownership rights and protection supporting the CSE practices have been observed from the literature. Consequently, the probability of potential legal pitfalls for both crowdsourcers and crowds might be increased while the popularity and demand for current and future CSE platforms might be reduced $[21,25]$. It is also important to note that controlling IP is not just constrained to concealing delicate data, as mentioned in the legal documents of CSE platforms, but can also be prolonged to other issues such as patents $[21,24,43]$.

\section{Conclusion}

This paper focuses on identification of CSE platforms and IP challenges encountered by the crowdsourcing users (crowdsourcers and crowds). The crowdsourcing approach requires some transfer of knowledge, tasks and work among the crowdsourcers and the crowds. As a result, the concern on the IP ownership rights and protection has become one of the most critical crowdsourcing challenges. We presented the crowdsourcing platforms that support both software engineering activities exclusively and partially and we presented the timeline of the establishment of these plat- 
forms within almost 2 decades from year 1998 to year 2015. Also, we classified these platforms into four categories based on the actual IP ownership created by the crowd either to the crowdsourcer, the crowd, the online platform, or unknown ownership. Analyses performed to 51 online platforms revealed that majority of the CSE platforms grant the IP ownership rights of crowdsourcing tasks to themselves. Also, there is lack of standard and guideline to govern IP ownership rights and exploitation that supports CSE activities. Consequently, future work shall establish a new guideline for the management and control of IP ownership rights and exploitation to help CSE platforms in drafting appropriate legal documents. Furthermore, we recommend to the crowdsourcers the performance of review of the legal documents of the platform to consider the suitability of the IP ownership rights option for their requested tasks. On the other hand, we recommend to the platforms the provision of different IP ownership options based on the probable circumstances of the crowdsourcers by considering the factors which can help in identifying each circumstances. This paper is expected to be beneficial to give an overview and generic guideline to the crowdsourcers and the crowds in choosing crowdsourcing platforms that suits their needs and to serve as a good starting point for future academic investigate.

\section{References}

[1] Stol, K. J., \& Fitzgerald, B. (2014). Two's company, three's a crowd: a case study of crowdsourcing software development. In Proceedings of the 36th International Conference on Software Engineering.2014 ISCE. Hyderabad, India. 31 May- 7 June. 187-198.

[2] Howe, J. (2006). The rise of crowdsourcing. Wired magazine, 14(6): 1-4.

[3] Geiger, D., Seedorf, S., Schulze, T., Nickerson, R. C., \& Schader, M. (2011, August). Managing the Crowd: Towards a Taxonomy of Crowdsourcing Processes. In AMCIS. 2011 proceeding of Seventh American Conference on information System. Deroit, Michigan. 4 7 August. 1-11.

[4] Stol, K. J., LaToza, T. D., \& Bird, C. (2017). Crowdsourcing for Software Engineering. IEEE Software, 34(2): 30-36.

[5] Sprugnoli, R., Moretti, G., Bentivogli, L., \& Giuliani, D. (2016) Creating a ground truth multilingual dataset of news and talk show transcriptions through crowdsourcing. Language Resources and Evaluation, 51(220): 1-35.

[6] Chatfield, A. T., \& Brajawidagda, U. (2014). Crowdsourcing hazardous weather reports from citizens via twittersphere under the short warning lead times of EF5 intensity tornado conditions. Paper presented at the 2014 47th Hawaii International Conference on System Sciences

[7] Brabham, D. C., Ribisl, K. M., Kirchner, T. R., \& Bernhardt, J. M. (2014). Crowdsourcing applications for public health. American journal of preventive medicine, 46(2): 179-187.

[8] Lakhani, K. R., \& Panetta, J. A. (2007). The principles of distributed innovation. innovations, 2(3): 97-112.

[9] Solemon, B., Ariffin, I., Din, M. M., \& Anwar, R. M. (2013). A review of the uses of crowdsourcing in higher education. International Journal of Asian Social Science, 3(9): 2066-2073

[10] Nag, S., Heffan, I., Saenz-Otero, A., \& Lydon, M. (2012, March). SPHERES Zero Robotics software development: Lessons on crowdsourcing and collaborative competition. IEEE Aerospace Conference 2012 Aerospace Conference. Big Sky, USA. 3-10 March. 1-17.

[11] Jeff, H. (2009). Crowdsourcing: why the power of the crowd is driving the future of business. Crown Business.

[12] LaToza, T. D., \& van der Hoek, A. (2016). Crowdsourcing in software engineering: models, opportunities, and challenges. IEEE Software. 33(1): 74-80.

[13] Archak, N. (2010). Money, glory and cheap talk: analyzing strategic behavior of contestants in simultaneous crowdsourcing contests on TopCoder. com. In Proceedings of the 19th international conference on World wide web, 2010 Raleigh. North Carolina, USA. 2630 April. 21-30.

[14] Begel, A., DeLine, R., \& Zimmermann, T. (2010). Social media for software engineering. In Proceedings of the FSE/SDP workshop on
Future of software engineering research, 2010 Foser. New Mexico, USA. 7-8 November. 33-38.

[15] Fitzgerald, B. (2012). Software Crisis 2.0. Computer, 45(4): 89-91.

[16] LaToza, T. D., Towne, W. B., Van Der Hoek, A., \& Herbsleb, J. D. (2013, May). Crowd development. In Cooperative and Human Aspects of Software Engineering (CHASE), 2013 6th International Workshop on. 2013 CHASE. San Francisco, USA. 85-88.

[17] Mao, K., Capra, L., Harman, M., \& Jia, Y. (2017). A survey of the use of crowdsourcing in software engineering. Journal of Systems and Software, 126: 57-84.

[18] Macmillan

Dictonary, http://dictionary.cambridge.org/dictionary/english/intellectualproperty (2016). Last access: 27/2/2014

[19] Neukom, W.H.: Intellectual Property in the New Technological Age. University of California at Berkeley. California (2016).

[20] Zhao, Y., Zhu, Q.: Evaluation on crowdsourcing research: Current status and future direction. J.Inf.Sys.Fron, 16, 417--434 (2014).

[21] Thuan, N. H., Antunes, P., \& Johnstone, D. (2016). Factors influencing the decision to crowdsource: A systematic literature review. Information Systems Frontiers, 18(1): 47-68.

[22] Khan, V. J., Dhillon, G., Piso, M., \& Schelle, K. (2016). Crowdsourcing user and design research. In Collaboration in Creative Design.121-148.

[23] Machado, L., Kroll, J., Prikladnicki, R., de Souza, C.R., Carmel, E.(2016). Software Crowdsourcing Challenges in the Brazilian IT Industry. In: 10th International Conference on Enterprice Information Systems. 2016 ICEIS.Rome, Italy. 25-28 April. 482--489.

[24] Burger-Helmchen, T., \& Pénin, J. (2010). The limits of crowdsourcing inventive activities: What do transaction cost theory and the evolutionary theories of the firm teach us. In Workshop on Open Source Innovation. 2010 AIMS. Strasbourg, France. 26 March. 1-26.

[25] Ford, R. C., Richard, B., \& Ciuchta, M. P. (2015). Crowdsourcing: A new way of employing non-employees?. Business Horizons, 58(4): 377-388.

[26] Afuah, A., Tucci, C.L.: Crowdsourcing as a solution to distant search. A.Man.Revi, 37, 355-375 (2012).

[27] Marjanovic, S., Fry, C., \& Chataway, J. (2012). Crowdsourcing based business models: In search of evidence for innovation 2.0. Science and Public Policy, 39(3): 318-332.

[28] Kannangara, S. N., \& Uguccioni, P. (2013). Risk management in crowdsourcing-based business ecosystems. Technology Innovation Management Review, 3(12): 23-38.

[29] Schenk, E., \& Guittard, C. (2011). Towards a characterization of crowdsourcing practices. Journal of Innovation Economics \& Management, 1(7): 93-107

[30] Ågerfalk, P. J., Fitzgerald, B., \& Stol, K. J. (2015). Software Sourcing in the Age of Open: Leveraging the Unknown Workforce. Springer.

[31] Machi, L. A., \& McEvoy, B. T. (2016). The literature review: Six steps to success: Corwin Press.

[32] Sajid, M., Al-bloush, H. B., Mohammed, A. F., Monsef, S., \& Sadeghi, M. (2015). Role of Innovation in the Development of New Products for Improving Organizational Performance. Journal of Advanced Management Science. 3(3): 261-264

[33] Oueslati, H., Rahman, M. M., \& ben Othmane, L. (2015). Literature review of the challenges of developing secure software using the agile approach. In Availability, Reliability and Security (ARES) 10th International Conference. 2015 ARES. Toulouse, France. 24 27 August.

[34] Mao, K., Yang, Y., Wang, Q., Jia, Y., \& Harman, M. (2015). Developer recommendation for crowdsourced software development tasks. In Service-Oriented System Engineering (SOSE), 2015 SOSE. San Francisco, USA. 30 March- 3 April. 347-356.

[35] Karger, D. R., Oh, S., \& Shah, D. (2014). Budget-optimal task allocation for reliable crowdsourcing systems. Operations Research, 62(1): 1-24.

[36] Boudreau, K. J., \& Lakhani, K. R. (2013). Using the crowd as an innovation partner. Harvard business review, 91(4): 60-69.

[37] Lohiya, R., John, P., \& Shah, P. (2015). Survey on Mobile Forensics. International Journal of Computer Applications, 118(16): 6-11.

[38] Claes, J., Vanderfeesten, I., Pinggera, J., Reijers, H. A., Weber, B., \& Poels, G. (2015). A visual analysis of the process of process modeling. Information Systems and e-Business Management, 13(1), 147-190.

[39] Kosinski, M., Bachrach, Y., Kasneci, G., Van-Gael, J., \& Graepel, T. (2012, June). Crowd iq: Measuring the intelligence of crowdsourcing platforms. In Proceedings of the 4th Annual ACM 
Web Science Conference Websci'12. New York, USA. 22-24 June. 151-160.

[40] Leeper, T. J. (2016). Crowdsourced data preprocessing with R and Amazon Mechanical Turk. The R Journal, 8(1): 276-288.

[41] Hasteer, N., Nazir, N., Bansal, A., \& Murthy, B. K. (2016) Crowdsourcing Software Development: Many Benefits Many Concerns. Procedia Computer Science, 78: 48-54

[42] World Intellectual Property Organization, IP ownership, http://www.wipo.int/sme/en/documents/wipo_magazine/11_2002.p df.

[43] Peng, X., Babar, M. A., \& Ebert, C. (2014). Collaborative software development platforms for crowdsourcing. IEEE software, 31(2): 30-36

[44] Bruun, A., \& Stage, J. (2015). New approaches to usability evaluation in software development: Barefoot and crowdsourcing. Journal of Systems and Software, 105: 40-53.

[45] Araujo, R. M. (2013). 99designs: An analysis of creative competition in crowdsourced design. In First AAAI conference on Human computation and crowdsourcing.2013 AAAI. California, USA. 7-9 November. 17-24.

[46] Li, H., Hao, L. Y., Ge, X., Gao, J., \& Guo, S. (2016). An agentbased approach for crowdsourcing software design. In Control and Decision Conference (CCDC), 2016 CCDC. Yinchuan, China. 28 30 May. 4497-4501.

[47] Hart, K., \& Sarma, A. (2014). Perceptions of answer quality in an online technical question and answer forum. In Proceedings of the 7th International Workshop on Cooperative and Human Aspects of Software Engineering. 2014 ACM. Hyderabad, India. 2-3 June. 103-106.

[48] Dubey, A., Abhinav, K., Taneja, S., Virdi, G., Dwarakanath, A., Kass, A., \& Kuriakose, M. S. (2016). Dynamics of software development crowdsourcing. In Global Software Engineering (ICGSE), 11th International Conference. 2016 ICGSE.Irvine, USA. 2-5 August. 49-58.

[49] Vukovic, M. (2009). Crowdsourcing for enterprises. In Services-I, World Conference. 2009, World Conference. Los Angeles, USA. 610 July. 686-692.

[50] Ambani, P. (2016). Crowdsourcing New Tools to Start Lean and Succeed in Entrepreneurship: Entrepreneurship in the Crowd Economy. Crowdfunding for Sustainable Entrepreneurship and Innovation,New Zeland.
[51] Lopez, M., Vukovic, M., \& Laredo, J. (2010). Peoplecloud service for enterprise crowdsourcing. In Services Computing (SCC), International Conference. 2010 SCC. Miami, USA. 5-10 July. 538-545.

[52] Costas, J., \& Grey, C. (2012). Outsourcing your Life: Exploitation and Exploration in "the 4-Hour Workweek". In Managing "Human Resources' by Exploiting and Exploring People's Potentials. 37 Emerals Group Puplishing Limited: 221-247.

[53] Oliveira, P. R. S. D. (2014). Crowdsourcing business models: focusing on the crowd-labor industry and the implications for management and markets. Doctoral dissertation

[54] Piao, C., Han, X., \& Jing, X. (2009). Research on web2. 0-based anti-cheating mechanism for witkey e-commerce. In Electronic Commerce and Security, 2009. ISECS'09.Nanchang, China. 22-24 March. 474-478

[55] Zogaj, S., Bretschneider, U., \& Leimeister, J. M. (2014). Managing crowdsourced software testing: a case study based insight on the challenges of a crowdsourcing intermediary. Journal of Business Economics, 84(3): 375-405

[56] Ruggieri, A., Mosconi, E. M., Poponi, S., \& Silvestri, C. (2016). Digital innovation in the job market: an explorative study on cloud working platforms. In Empowering Organizations. 11: 273-283.

[57] Hong, Y., \& Pavlou, P. A. (2013). Online labor markets: An informal freelancer economy.

[58] Alsmadi, I., \& Saeed, S. (2013). A software development process for open source and open competition projects. International Journal of Business Information Systems, 12(1): 110-122.

[59] Munoz-Chacon, D. (2012). Creating a Women Business Owners Online Membership Site Using The 24-Hour Knowledge Factory.

[60] Gupta, A., Thies, W., Cutrell, E., \& Balakrishnan, R. (2012). mClerk: enabling mobile crowdsourcing in developing regions. In Proceedings of the SIGCHI Conference on Human Factors in Computing Systems. 2012 SIGCHI. Texas, USA. 5-10 May. 1843-1852.

[61] To, W. M., \& Lai, L. S. (2015). Crowdsourcing in China: Opportunities and concerns. IT Professional, 17(3): 53-59.

[62] Brandon, D. M., Long, J. H., Loraas, T. M., Mueller-Phillips, J., \& Vansant, B. (2013). Online instrument delivery and participant recruitment services: Emerging opportunities for behavioral accounting research. Behavioral Research in Accounting, 26(1): 1-23.

[63] Peng, X., Gu, J., Tan, T. H., Sun, J., Yu, Y., Nuseibeh, B., \& Zhao, W. (2016). CrowdService: Serving the individuals through mobile crowdsourcing and service composition. Automated Software Engineering (ASE.). 31st IEEE/ACM International Conference. 2016ASE. Singapore, Singapore. 3-7 September. 214-219.

\begin{tabular}{|c|c|c|c|c|}
\hline ID & Name & URL & Primary Work Category & Ref \\
\hline $\mathrm{P} 1$ & TopCoder & http://www.topcoder.com/ & Software Development & {$[1]$} \\
\hline $\mathrm{P} 2$ & GetACoder & http://www.getacoder.com/ & Software Development & [34] \\
\hline P3 & Innocentive & http://www.innocentive.com/ & Problem Solving & {$[17]$} \\
\hline $\mathrm{P} 4$ & Geniusrocket & http://geniusrocket.com/ & \begin{tabular}{|l|} 
Graphic Design \\
\end{tabular} & {$[43]$} \\
\hline P5 & TryMyUI & http://www.trymyui.com/ & Software Testing & {$[44]$} \\
\hline P6 & Usertesting & http://www.usertesting.com/ & Software Testing & [44] \\
\hline $\mathrm{P} 7$ & 99designs & http://99designs.com/ & Graphic Design & {$[45]$} \\
\hline P8 & uTest & http://www.utest.com/ & Software Testing & {$[46]$} \\
\hline $\mathrm{P9}$ & Stackoverflow & http://stackoverflow.com/ & Software Development & {$[47]$} \\
\hline $\mathrm{P} 10$ & Passbrains & http://www.passbrains.com/ & Software Testing & {$[48]$} \\
\hline $\mathrm{P} 11$ & \begin{tabular}{|l|} 
99Tests \\
\end{tabular} & http://www.99tests.com/ & Software Testing & {$[17]$} \\
\hline $\mathrm{P} 12$ & TestBirds & http://www.testbirds.com/ & Software Testing & {$[48]$} \\
\hline P13 & TestBats & http://www.testbats.com/ & Software Testing & {$[12]$} \\
\hline P14 & Pay4Bugs & http://www.pay4bugs.com/ & Software Testing & {$[12]$} \\
\hline P15 & CrowdTesters & http://www.crowdtesters.com.au/ & Software Testing & {$[17]$} \\
\hline P16 & TestFlight & http://www.testflightapp.com/ & Mobile App Testing & {$[46]$} \\
\hline P17 & Mob4hire & http://www.mob4hire.com/ & Mobile App Testing & {$[17]$} \\
\hline P18 & Testin & http://www.itestin.com/ & Mobile App Testing & {$[17]$} \\
\hline P19 & Ce.WooYun & http://ce.wooyun.org/ & Software Security Testing & {$[17]$} \\
\hline $\mathrm{P} 20$ & Bugcrowd & http://www.bugcrowd.com/ & Software Security Testing & {$[12]$} \\
\hline $\mathrm{P} 21$ & Guru & http://www.theknowledgeguru.com/ & Software Security Testing & {$[48]$} \\
\hline $\mathrm{P} 22$ & Freelancer & http://www.freelancer.com/ & \begin{tabular}{|l|} 
Project Marketplace \\
\end{tabular} & {$[25]$} \\
\hline $\mathrm{P} 23$ & Tacken & Not found & & {$[34]$} \\
\hline P24 & Upwork & https://www.upwork.com/ & Project Marketplace & {$[48]$} \\
\hline $\mathrm{P} 25$ & AMT & http://www.mturk.com/ & Project Marketplace & {$[17]$} \\
\hline P26 & Fiverr & https://www.fiverr.com/ & Project Marketplace & {$[38]$} \\
\hline P27 & Crowdflower & http://www.crowdflower.com/ & Data Mining & {$[17]$} \\
\hline $\mathrm{P} 28$ & Askville-Amaz & http://askville.amazon.com/ & Question-and-Answer & {$[21]$} \\
\hline P29 & PeoplePerHour & http://www.peopleperhour.com/ & Project Marketplace & {$[49]$} \\
\hline $\mathrm{P} 30$ & Crowdspirit & http://www.crowdsourcing.org/ & Graphic Design & {$[25]$} \\
\hline P31 & GetSatisfaction & https://getsatisfaction.com/ & Technical Support & {$[50]$} \\
\hline
\end{tabular}




\begin{tabular}{|l|l|l|l|}
\hline P32 & Fixya & http://www.fixya.com/ & Question-and-Answer \\
\hline P33 & Getfriday & https://getfriday.com/ & Project Marketplace \\
\hline P34 & BizReef & http://www.bizreef.com/ & Project Marketplace \\
\hline P35 & CGILance.com & http://www.cqilance.com/ & Programmer Marketplace \\
\hline P36 & Chaordix Inc. & http://www.chaordix.com/ & Problem Solving \\
\hline P37 & Eufreelance & http://www.eufreelance.com/ & Software Development \\
\hline P38 & Freelance Web Program & http://www.freelancewebprogram.com/ & Programmer Marketplace \\
\hline P39 & Limeexchange & http://www.limeexchange.com/ & [53] \\
\hline P40 & Programmibids & http://www.programmingbids.com/ & Software Development \\
\hline P41 & Rent A Coder & http://www.rentacoder.com/ & Programmer Marketplace \\
\hline P42 & Scriptlance & http://www.scriptlance.com/ & Software Development \\
\hline P43 & DesignQuote & http://www.designquote.net/ & Software Development \\
\hline P44 & Programmermeetdesigner & http://www.programmermeetdesi.com/ & Graphic Design \\
\hline P45 & Project4hire & www.project4hire.com & Software Development \\
\hline P46 & LiveWork & http://www.livework.com/ & Project Marketplace \\
\hline P47 & MobileWorks & http://www.mobileworks.com/ & Any Tasks \\
\hline P48 & Witmart & http://www.witmart.com/ & Any Tasks \\
\hline P49 & CrowdSpring & http://www.crowdspring.com/ & [58] \\
\hline P50 & Zintro & http://www.zintro.com/ & Project Marketplace \\
\hline P51 & AppStori & http://www.appstori.com/ & Graphic Design \\
\hline
\end{tabular}

